\title{
Scanning of a Double-Sided Germanium Strip Detector
}

\author{
Arzoo Sharma ${ }^{1,3}$, R. Palit ${ }^{2}$, I. Kojouharov ${ }^{3}$, J. Gerl ${ }^{3}$, M. Gorska-Ott ${ }^{3}$, H. Schaffner ${ }^{3}$, T. Habermann ${ }^{3}$, \\ S. Saha ${ }^{4}$, Biswajit Das ${ }^{2}$, P. Dey ${ }^{2}$, R. Donthi ${ }^{2}$, B.S. Naidu ${ }^{2}$, S. Mandal ${ }^{5}$, Pushpendra P. Singh ${ }^{1}$ \\ ${ }^{1}$ Department of Physics, Indian Institute of Technology Ropar, Rupnagar - 140 001, Punjab, India \\ ${ }^{2}$ Department of Nuclear and Atomic Physics, Tata Institute of Fundamental Research, \\ Homi Bhabha Road, Mumbai - 400 005, India \\ ${ }^{3}$ GSI Helmholtzzentrum für Schwerionenforschung GmbH, Planckstrasse 1, 64291 Darmstadt, Germany \\ ${ }^{4}$ University of Massachusetts Lowell, Lowell, Massachusetts 01854, USA \\ ${ }^{5}$ Department of Physics, North Campus, University of Delhi, Delhi - 110 007, India \\ palit@tifr.res.in, 2017phz0005@iitrpr.ac.in
}

\begin{abstract}
This work presents the results from the characterization of a Position-Sensitive Planar Germanium (PSPGe) detector. The PSPGe detector is a double-sided orthogonal strip detector consisting of 10x10 electrical segmentation along the horizontal and vertical directions. The characterization was performed using the coincidence setup between the PSPGe detector and the well-characterized scanning system employing the positron annihilation correlation principle. The scanning system consists of a Position Sensitive Detector (PSD) and ${ }^{22} \mathrm{Na}$ positron source. The main objective of this study is to deploy PSPGe detector for future decay experiments at the Facility for Antiproton and Ion Research (FAIR), Germany. The measurements have been performed to find the depth of gamma-ray interaction in the planar segmented detector. The 2Dimensional image obtained from the PSD has been used to find the depth of gamma-ray interaction in the planar strip detector using pulse shape analysis. In addition, the sensitivity of PSPGe detector has been investigated by calculating the rise-time from pulse shapes for the front and back strips of the detector.
\end{abstract}

Keywords -Double-Sided Strip Detector; Positron Annihilation Correlation; Position Sensitive Detector; Pulse Shape Analysis.

\section{INTRODUCTION}

$\mathrm{W}$ HILE the nucleus of an atom is a complex object; it has been studied extensively with the aid of characteristic gamma-ray emitted from its decay using gamma spectroscopy tools. The understanding of the structure of nuclei has been obtained through the development of sophisticated solid-state detectors. An advent in this field has been through the development of highly segmented High Purity Germanium (HPGe) detectors like AGATA [1] and GRETA [2]. These detectors are employed to track the path of gamma-ray interaction inside the detector crystal using energy, time, and location information. They are coaxial $4 \pi$ HPGe detector arrays based on the principle of Pulse Shape Analysis (PSA) [3]. The pulses are stored for each segmented crystal within the defined time window of supporting electronics. Various PSA algorithms have been employed to locate the interaction position that is based on the principle of the Shockley-Ramo theorem [4]. It states that the moving charge, produced by gamma-ray interaction, induces a charge on the electrode, which is detected by the charge-sensitive pre-amplifiers. The correct PSA algorithm helps to reconstruct the complete path of a photon interacting at various locations using the Compton scattering formula. If the algorithm is not reasonably constructed, it may lead to the complete loss of several interacting events. This may further provide wrong energy. Hence, the reasonable choice of PSA algorithm has to be made depending upon the detector geometry, electrode, and segmentation. Therefore, the complexity of signals in these segmented detectors requires complete characterization with known gamma-ray sources.

This manuscript has been put forth to characterize a Planar Position-Sensitive Germanium (PSPGe) detector along the depth. The detector depth is not segmented. Hence, the risetime studies have been performed to find depth of gamma-ray interaction [5]. Further, the detector has been scanned using a scanning facility developed at GSI Helmholtz Centre for heavyion research, Germany $[6,7]$. The main aim of performing these tests is to employ the detector as an implantation detector in the Decay SPECtroscopy (DESPEC) experiments in the framework of Facility for Antiproton and Ion Research (FAIR) at GSI [8]. This article is organized as follows. Section II describes the detailed experimental setup. In section III, the analysis procedure is discussed. The Results and conclusions are presented in sections IV and V.

\section{EXPERIMENTAL DETAILS}

The PSPGe detector is a p-type electrically segmented detector. It consists of 10 segmentation along both horizontal as well as vertical directions. The horizontal strips are DC coupled, and the vertical strips are AC coupled. The detector has dimensions of $6 \times 6 \times 2 \mathrm{~cm}^{3}$. The energy resolution and efficiency details of the detector are described in the reference [9]. The DC electrodes are etched on the front side of the detector, while AC strips are at the backside. In the present work, DC and AC electrodes are referred to as DC0 to DC9, 
and AC16 to AC25, respectively, as indicated in figure 1.

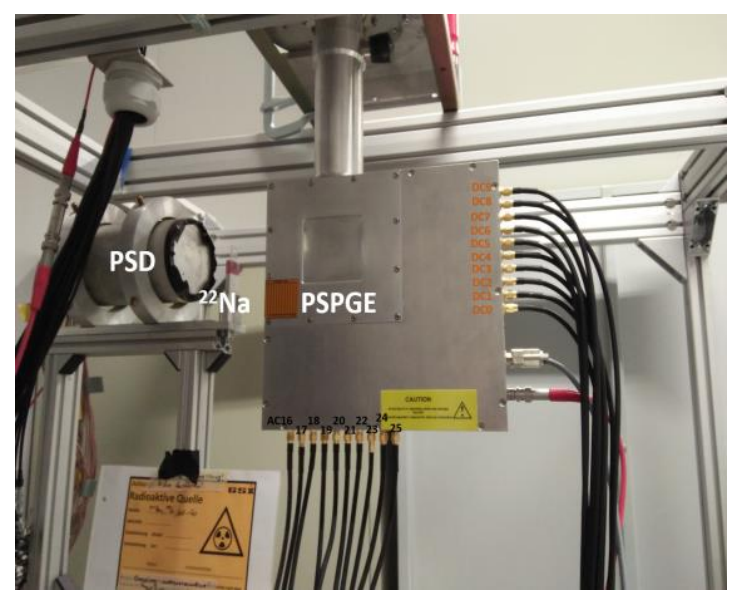

Fig. 1: Typical coincidence setup between PSD (left) and PSPGe detector (right) (DC and AC strips are marked in orange and blue color, respectively).

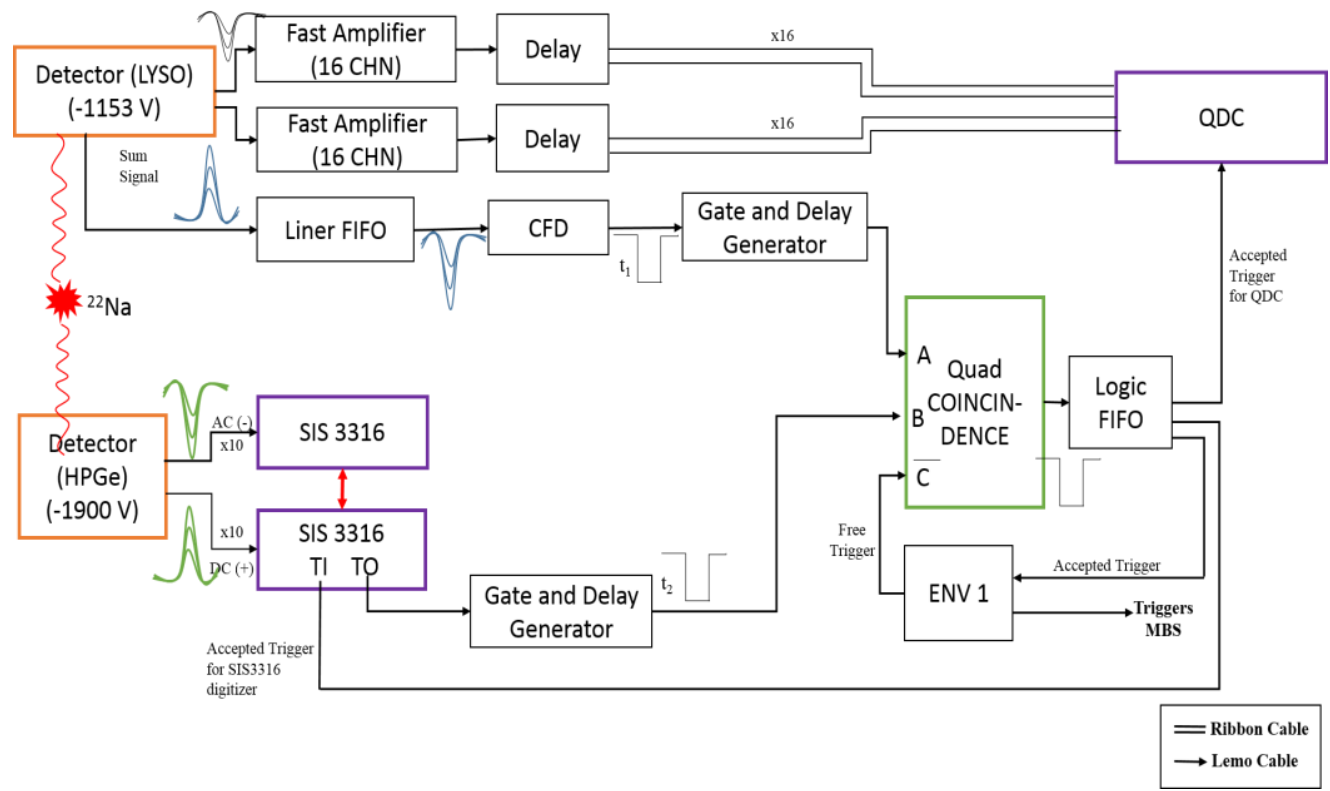

Fig. 2: Typical block diagram of electronics arrangement used for setting up the coincidence between PSD and PSPGe detectors.

The experimental setup is shown in Fig. 1. The PSPGe detector has been set up in coincidence with the PSD using ${ }^{22} \mathrm{Na}$ source. The PSD consists of a LYSO scintillator coupled to Position-Sensitive Photo Multiplier Tube (PSPMT) [10]. After pair annihilation, the two gamma-rays are detected in PSPGe and PSD. The distance between ${ }^{22} \mathrm{Na}$ source and PSPGe detector was kept $8.6 \mathrm{~cm}$. The distance was optimized to get a complete image by looking at the online 2-Dimensional (2-D) image using GSI Object Oriented On-line Off-line (GO4) software. The 2-D image is extracted from PSD, consisting of PSPMT with 16-X and 16-Y anodes mesh. The complete electronics block diagram is shown in Fig. 2. The 32 anode output signals from the PSD were amplified using Fast Amplifier (N979). Afterward, the output was fed to Versa Module Eurocard (VME) based multi-event Charge to Digital Convertor (QDC V792) unit with 12-bit resolution. The cathode output from the PSD was used to generate timing gate, $\mathrm{t}_{1}$ (depicted in Fig. 2), via Constant Fraction Discriminator (CFD - CF4000). The 10 DC and 10 AC outputs of the PSPGe detector were fed to $100 \mathrm{MHz}$ digitizers from Struck Innovative Systeme (SIS3316) modules. It provides information on pulse shape and energy for each interacting gamma-ray on an eventby-event basis. The digitizer's Trigger-Out (TO) output was a logic pulse that contained information of the OR (OR of the time) of all channels, indicated as time $t_{2}$ in Fig. 2. Afterward, $t_{1}$ and $t_{2}$ were set up in coincidence using the Quad Coincidence unit (CO4001). The data from QDC and SIS3316 was read out using the Multi-Branching Data Acquisition System (MBS). The ENV1 module contains the dead time of the MBS readout, which is put in anti-coincidence with $t_{1}$ and $t_{2}$ using CO4001. The output was used as an accepted trigger for the QDC and SIS3316 digitizer. The settings of the digitizer were made using desktop Graphical User Interface (GUI). The monitored data rate was $\sim 250 \mathrm{~Hz}$. 


\section{DATA ANALYSIS PROCEDURE}

The data analysis has been performed using GO4, C++, and ROOT. The critical points of the analysis procedure are listed here. First, the 2-D image is reconstructed from the charge collected at X and $\mathrm{Y}$ anodes in PSD for the coincidence events. The reconstruction has been obtained by the centroid fitting method, as displayed in Fig. 3.

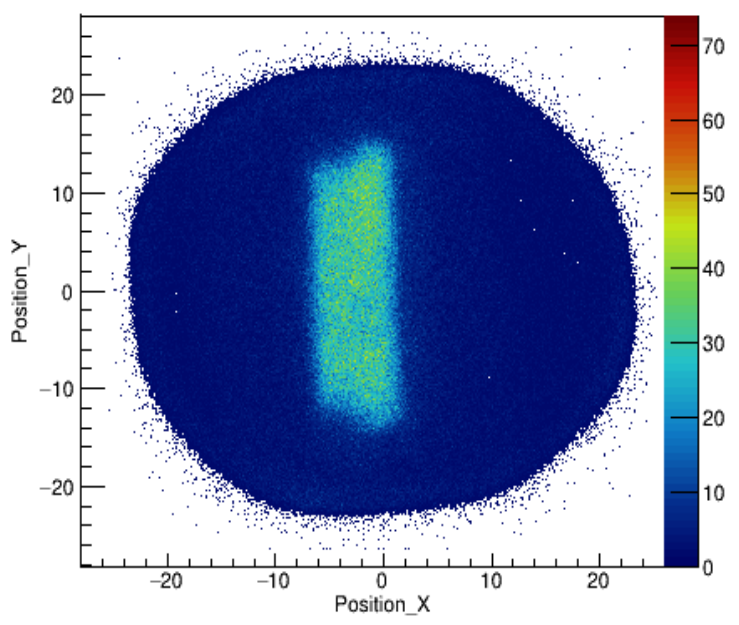

Fig. 3: The 2-D image obtained from PSD.

The middle region with maximum counts represents the active region of detector volume. 2-D image is projected onto $\mathrm{X}$-axis, and it represents the depth of PSPGe. It is used for dividing the depth into segments because of no segmentation along the depth. First, the obtained energy spectra from the digitizer are calibrated. Then, it is used to select an electrode of interest by gating on the Compton edge. The pulse shape and/or trace obtained from the digitizer provides information on the gammainteraction event for the selected electrode. The analysis has been performed for the obtained traces as described in reference [11], i.e., the baseline subtraction is performed, followed by normalization. Afterward, traces are time-aligned at $10 \%$ of the final amplitude.

The work has been presented to understand the analysis for finding the depth of gamma-ray interaction using the setup shown in Fig. 1. The Compton edge of $511 \mathrm{keV}$ photopeak is used to select two middle strips DC4 and AC20. The pulse shape is analyzed for different points along the depth. The rise time is measured for selected traces at a specific depth location. The average of multiple traces is considered to find the risetime. The sensitivity of the PSPGe detector towards depth would depend on how precisely charge collection could be measured for two charge carriers (electrons and holes) at the two opposite-facing electrodes. It would then lead to different rise-time values at different depth locations. In the scope of the present analysis, T30 rise-times have been calculated for DC4 and AC20 strips. It is defined as the time required to reach $30 \%$ of the maximum amplitude minus the time required to reach $10 \%$ of the maximum amplitude. The analysis results, for the behavior of both DC and AC strips, have been discussed in detail in the upcoming section.

\section{RESULTS AND DISCUSSION}

The coincidence technique using the principle of positron annihilation has been used to characterize the electrically segmented PSPGe detector. The increased segmentation is beneficial to track each Compton scattered event in a different segment. The centroid fitting method has been used to reconstruct the image from PSD.

To understand the charge induced by the incoming gammarays, the rise-time has been calculated for the pulse shapes obtained from SIS3316 digitizer. The analysis has been performed using energy cut at Compton edge for two middle strips, DC4 and AC20. As a representative case, the behavior of DC4 and AC20 strips is shown in Fig. 4, for one of the middle cuts. In the figure, the amplitude of the trace is plotted as a function of time. The obtained T30 rise-time values for DC4 and AC20 strips are $30 \mathrm{~ns}$ and $80 \mathrm{~ns}$, respectively. This shows the appreciable time difference between the two traces. This feature can be further used to find the rise-time as a function of depth.

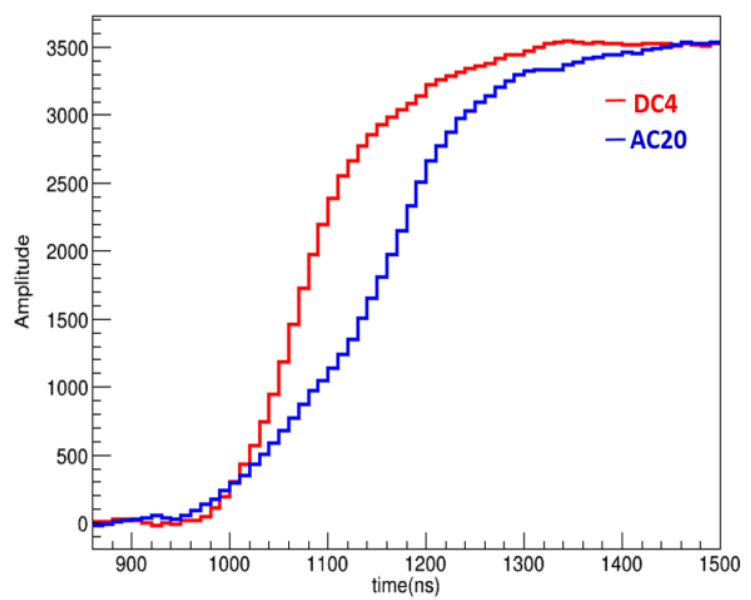

Fig. 4: T10 aligned traces for a middle cut selection $(-2.1<$ Position_X<-1.9) on active region in 2-D image obtained from PSD. Traces of DC4 and AC20 are represented by red and blue color, respectively.

The difference in rise-times of the two strips may be attributed to the movement of charge carriers towards the respective electrodes. The front face of the detector consists of DC segments, and the AC segments are etched on the backside of the detector. The electrons would travel towards the DC electrode while holes would be collected at the AC electrode. The rise-time distribution is plotted for one of the depths in Fig. 5. In this plot, T30 rise-time counts are represented by a black histogram. The red curve is the Gaussian fit to find the centroid of the distribution. Hence, the centroid can be used to find the rise-time at a specific depth. In addition, the spread in the distribution may be used to infer the error in the obtained risetime values [12]. 


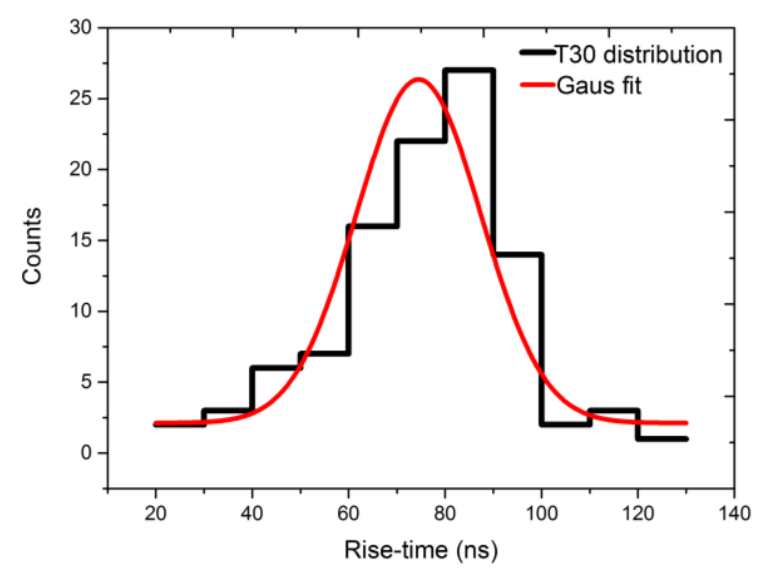

Fig. 5: Plot of counts vs rise-time (T30) at a specific depth. The red curve is the Gaussian fit (used to find centroid of the distribution).

The analysis has been extended to find the rise-time as a function of depth for both the DC4 and AC20 electrodes. It is shown in Fig. 6(a)-(b).
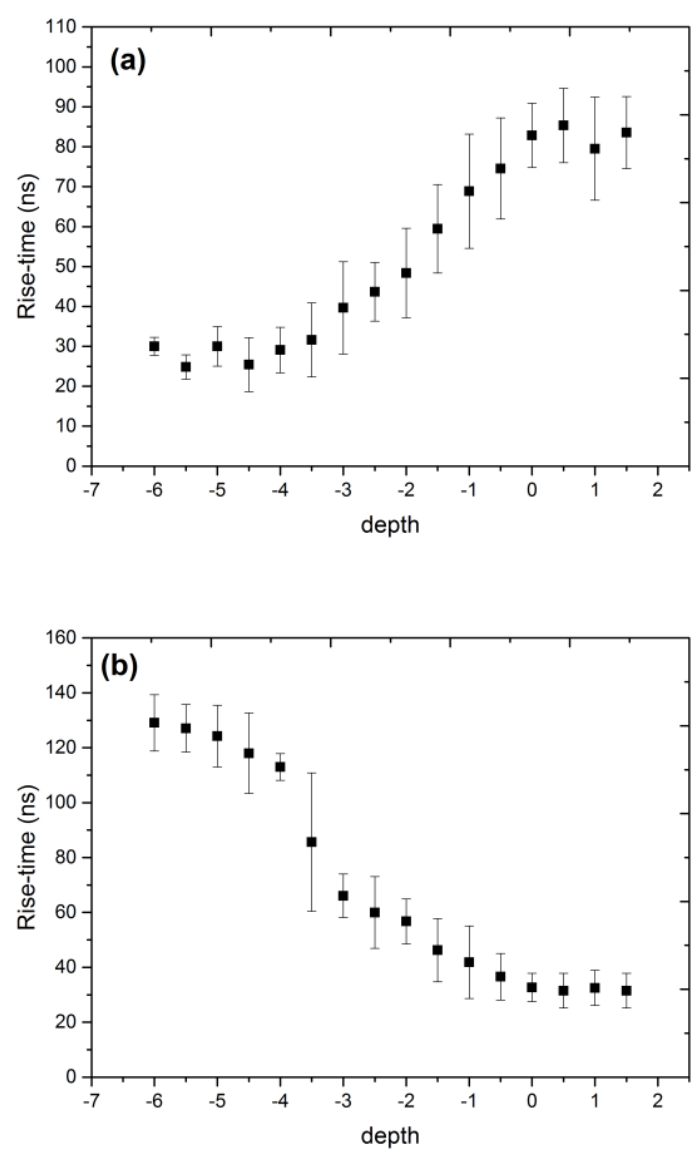

Fig. 6: T30 rise-time values as a function of depth for (a) DC4 and (b) AC20 segments, respectively.

In this figure, $\mathrm{X}$-axis represents depth, i.e., going from the front to the backside of the detector. The values plotted are the centroids of the rise-time distribution obtained for each depth. The error has been calculated for each data point from the risetime distribution spread, as indicated in Fig. 5. For DC4 (Fig. 6(a)), with an increase in depth, rise-time increases. Fig. 6(b) shows that the rise-time of AC20 decreases with an increase in depth. The rise-time of the DC4 segment starts to increase while traversing from the left to right side, i.e., from DC towards the AC electrodes. It occurs because of the slow collection of electrons at the front surface with an increase in depth.

In comparison, the holes are collected faster, therefore, the faster rise-time response at the AC electrode. This implies that the difference in collection times of the two charge carriers, between the front and backside of the detector, can successfully be applied to find the depth of the gamma-ray interaction. From the above analysis, it may be deduced that the position response of the detector is sensitive towards charge collection at the respective electrodes.

\section{SUMMARY AND CONCLUSIONS}

In summary, a PSPGe detector has been characterized using a time-efficient scanning facility at GSI Germany. The response of the detector has been studied for gamma-ray interaction along the depth using rise-time values obtained from the pulse shape analysis. The behavior of induced charge along the detector surface has been obtained and analyzed using rise-time calculation as a function of depth. The T30 rise-time distribution has been used to find the position sensitivity of the detector towards a gamma-ray hit. The results obtained in this work have been promising for finding the position sensitivity of the detector as the subsequent analysis steps.

\section{ACKNOWLEDGMENT}

This work is partially supported by the Department of Atomic Energy, Government of India, under project No. 12P-R\&DTFR-5.02-0400. The authors would like to acknowledge the Helmholtz International Center for FAIR in Germany and Prof. H. J. Wollersheim for constant support for carrying out this work.

\section{REFERENCES}

[1] S. Akkoyun, A. Algora, B. Alikhani, F. Ameil, G. De Angelis, L. Arnold, A. Astier, A. Ataç, Y. Aubert, and C. Aufranc, "Agata-advanced gamma tracking array". Nuclear Instruments Methods in Physics Research Section A: Accelerators, Spectrometers, Detectors Associated Equipment 2012. 668: p. 26-58. K. Vetter, A. Kuhn, I. Lee, R. Clark, M. Cromaz, M. Deleplanque, R. Diamond, P. Fallon, G. Lane, and A. Macchiavelli, "Performance of the GRETA prototype detectors". Nuclear Instruments Methods in Physics Research Section A: Accelerators, Spectrometers, Detectors Associated Equipment 2000. 452(1-2): p. 105-114.

[3] F. Crespi, F. Camera, B. Million, M. Sassi, O. Wieland, and A. Bracco, "A novel technique for the characterization of a HPGe detector response based on pulse shape comparison". Nuclear Instruments Methods in Physics Research Section A: Accelerators, Spectrometers, Detectors Associated Equipment, 2008. 593(3): p. 440-447.

[4] Z. He, "Review of the Shockley-Ramo theorem and its application in semiconductor gamma-ray detectors". 
Nuclear Instruments Methods in Physics Research Section A: Accelerators, Spectrometers, Detectors Associated Equipment 2001. 463(1-2): p. 250-267.

[5] M. Momayezi, W. K. Warburton, and R. A. Kroeger. "Position resolution in a Ge-strip detector". in Hard X-Ray, Gamma-Ray, and Neutron Detector Physics. 1999. International Society for Optics and Photonics.

[6] T. Habermann, F. Didierjean, G. Duchêne, M. Filliger, J. Gerl, I. Kojouharov, G. Li, N. Pietralla, H. Schaffner, and M.-H. Sigward, "Application of gamma imaging techniques for the characterisation of position sensitive gamma detectors". Nuclear Instruments Methods in Physics Research Section A: Accelerators, Spectrometers, Detectors Associated Equipment, 2017. 873: p. 24-26.

[7] C. Domingo-Pardo, N. Goel, T. Engert, J. Gerl, I. Kojouharov, H. Schaffner, F. Didierjean, G. Duchêne, and M. Sigward, "A novel $\gamma$-ray imaging method for the pulse-shape characterization of position sensitive semiconductor radiation detectors". Nuclear Instruments Methods in Physics Research Section A: Accelerators, Spectrometers, Detectors Associated Equipment, 2011. 643(1): p. 79-88.

[8] A. Khaplanov, B. Cederwall, and S. Tashenov, "Position sensitivity of segmented planar HPGe detectors for the DESPEC project at FAIR". Nuclear Instruments Methods in Physics Research Section A: Accelerators, Spectrometers, Detectors Associated Equipment, 2008. 592(3): p. 325-333.

[9] J. Sethi, R. Palit, B. Naidu, S. Saha, T. Trivedi, R. Donthi, and S. Jadhav. "Characterization of a Planar Ge Strip Detector and its Coupling with a Segmented HPGe Clover Detector". in Proceedings of the DAE Symp. on Nucl. Phys. 2011.

[10] N. Goel, C. Domingo-Pardo, T. Engert, J. Gerl, I. Kojouharov, and H. Schaffner, "Spatial calibration via imaging techniques of a novel scanning system for the pulse shape characterisation of position sensitive HPGe detectors". Nuclear Instruments Methods in Physics Research Section A: Accelerators, Spectrometers, Detectors Associated Equipment, 2011. 652(1): p. 591-594.

[11] N. Goel, C. Domingo-Pardo, T. Habermann, F. Ameil, T. Engert, J. Gerl, I. Kojouharov, J. Maruhn, N. Pietralla, and H. Schaffner, "Characterisation of a symmetric AGATA detector using the $\gamma$-ray imaging scanning technique". Nuclear Instruments Methods in Physics Research Section A: Accelerators, Spectrometers, Detectors Associated Equipment, 2013. 700: p. 10-21.

[12] M. Descovich, P. Nolan, A. Boston, J. Dobson, S. Gros, J. Cresswell, J. Simpson, I. Lazarus, P. Regan, and J. Valiente-Dobon, "The position response of a large-volume segmented germanium detector". Nuclear Instruments Methods in Physics Research Section A: Accelerators, Spectrometers, Detectors Associated Equipment, 2005. 553(3): p. 512-521. 